\title{
UNDERSTANDING THE USER WITHIN THE INNOVATION SPIRAL
}

\author{
Reginald Coutts ${ }^{1}$, Pamela Coutts ${ }^{2}$ and Kate Alport ${ }^{1}$ \\ ${ }^{I}$ Coutts Communications, 30-32 Stirling St, Thebarton, SA 5031 and University of Adelaide, \\ Nth Terrace Adelaide $S A ;{ }^{2}$ Coutts Communications;
}

Abstract: This paper will examine the concept of an innovation spiral process in relationship to the mobile communications sector of the Information and Communications Technology (ICT) industry which is the product of convergence of the telecommunications and the information technology (IT) industries. The fundamental theoretical framework for the paper is that innovative applications prescribed by users of an adopted technology can be a significant driver of further product evolution which then can fuel further market innovation. New products that are spectacularly 'successful' are those that give rise to this spiral of product innovation and market innovation. The paper will tirst review the macro perspective of this theoretical framework by analysing the spectacular success of the short message service (SMS) in the GSM digital mobile communications over the last twenty years from the product concept. This conceptual framework is then considered at a micro level to review three consecutive research projects by the authors over the last ten years. The broad aim of these projects was to better understand potential user adeption of new mobile telecommunications products. The first researci project in the mid 1990's examined the barriers and enablers to the adoption of mobile phones by selected disadvantaged groups in society, in particular, people with disabilities. A modified focus group methodology based on interactive workshops was developed from this research project to gain insights into user innovation. This methodology was developed further in more recent research projects looking at the likely user take up of evolving multimedia capable mobile devices for innovative applications. The second study indicated that the market evolution of mobile internet like applications is likely to be very different from those developed for the fixed internet because of the different characteristics of the user groups. While the individual research projects have been published, this paper brings together the macro and micro perspectives of this innovation spiral to demonstrate the value of this theoretical framework for market forecasting for realising technology commercialisation. The research also has implications for the 'form' of new 
high technology products and how they are marketed which places less emphasis on technical features but more on matching with user needs.

Keywords: innovation; telecommunications; mobile communications; GSM; SMS; qualitative research; multimedia; commercialization

\section{INTRODUCTION}

There has been much written about 'disruptive technologies' (Christensen, 1996) in the last nine years particularly with reference to the dramatic effects of innovations in ICT technologies which have reshaped economies, companies and the way people live. It is clear that new perspectives on how innovation evolves are still required to give deeper insight into how to better-forecast future technology adoption scenarios.

Bar has described the evolutionary nature of the innovation process that "highlights the existence of feedback loops, interactions and linkages between users and producers" (Bar and Riis 2000). For complex network based service products like in the telecommunications sector, Damsgaard develops a new framework combining technology evolution and service adoption that recognises the distinct but interactive role of infrastructure and service innovation described as "A self-enforcing spiral of mutual reenforcement of both infrastructure innovation and innovation adoption can unfold under favourable circumstances" (Damsgaard and Gao 2004). This paper extends this framework to consider distinct phases of innovation.

While this paper does not pretend tc offer the answers to the forecasting of the market or user demand for ICT technologies, it does offer a new perspective on how innovation gathers pace in distinct phases and the key role of users in this process. The process in each of these phases has a different relative dynamic of market and product innovation which we call the innovation spiral.

To illustrate the conceptual utility of this evolution framework, the paper considers the history of the evolution of the SMS service in GSM that is generally regarded as one of the most successful innovations in the mobile sector of telecommunications in the last ten years. The evolution of the product and the market are seen as occurring in four phases. The 'innovation spiral' as we have termed to describe the dynamic has distinct characteristics in each different phase. This could be considered the 'macro' view of this innovation evolution process.

The paper then examines three successive user focused research projects by the authors (Coutts, 1998, 2002, 2003) conducted over the last seven years in the context of this conceptual framework for understanding the lay 
users role in mobile technology evolution. It has been noted that lay users "often think outside of the box and imagine potential applications that are not simply extensions of existing practices" (Bar and Riis 2000). Evidence suggests that the "tight relationships between users and producers from early stages of research are especially critical where radical innovation is concerned" (Bar and Riis 2000). The methodology developed during this research in a sense provides a window into how users appropriate and drive innovation. Experience with a modified focus group methodology that significantly improves the ability to develop insights into how the user innovates, can indicate the likely developmental phases of a technology. We define this process as user innovation in that it inspires innovation by the industry to modify the product or create a new one!

The last part of this paper brings the two macro and micro perspectives together to illustrate the utility of the innovation spiral and a methodology in developing more informed scenarios of future demand for ICT product innovations. The overall recommendation arising from this research and the proposed framework is for operators to undertake regular engagement with the lay user community in order to monitor and in a sense calibrate the innovation evolution of the range of products.

\section{TECHNOLOGY INNOVATION FRAMEWORK}

The conceptual framework to be developed in this paper is that successful technology innovation can be view'ed as going through distinct phases where at one or more phases the 'innovation spirals' when the market rapidly expands in an 'unpredicrable way'. This framework builds on the concept of a "self-enforcing spiral" coined by Damsgaard (Damsgaard and Gao 2004) but with the aim of gaining insights from lay users about how the technology may evolve.

In the initial phase, at the onset of innovation, there is both market and product uncertainty (Utterback et al 1995) and is the point before any innovation spiral, as we define it can begin to occur or turn. The phases of interactive innovation start when the service is available to users.

For the innovation spiral to occur, users or as it is otherwise termed, a market segment of a technology redefine the utility of the technology through experience, giving rise to further technology improvement or even giving rise to a new variant of the technology. This user inspired innovation fuels even greater user adoption. The innovation spiralling effect can accelerate in distinct phases in the product lifecycle that results in what 'appears' to be a discontinuity. In reality the change is continuous, even 
though adoption occurs at a different rate. The challenge of the developed modified focus group approach discussed in section 4 is to gain key insights into these potential inflexion points of increased innovation and market adoption.

Firstly, it is necessary to define our terms. Technology can be understood as "the knowledge embodied in human action to achieve practical results". Invention is the creation of a new idea or concept whereas innovation is the process of turning the new concept into commercial success or widespread use. This paper is primarily concerned with technological innovations which are those with a significant "performance content". However, such 'performance content' must be able to be realised in increased market value.

The proposed hypothesis is that users of technology can reinterpret the utility of a technology considered in a way that the industry does not anticipate and that an understanding of that process can inform market evolution forecasting. This 'innovation spiral' has been examined in the context of technologies in the mobile sector of the telecommunications industry with reference to the history of the short messaging service (SMS) on mobile phones. In the case of the history of SMS, the market or user experience of the early form of SMS spurred a response, albeit rather belatedly, by the industry to explore and develop the technological and commercial means of providing an improved capability. It is argued that $n$ this way the users are functioning as innovators which then promotes an innovative technological response from the industry. In this spiralling pattern of innovation the user is doing much more than just being stimulated to buy and use a new service, they are actually adapting the existing technology and effectiveiy creating a new technology. In a sense this series of innovation spirals and can be considered as distinct re-tuning of the familiar market adoption $S$ curve as the product better meets market demand.

This process of users reinterpreting or creating a new context for a communications technology platform was first observed by the authors (Coutts 1998) in their research with workers in the community service sector supporting people with disabilities. Despite the assumed high utility of a mobile service for a workforce that was undergoing decentralisation and increasingly working out of the office under time pressure, take up of mobiles was very low. In their work with this sector the researchers were in fact promoting and observing the process of user innovation - a process that usually takes place unobserved and unscripted, unchoreographed or recorded, let alone interpreted, in a way that would ultimately make it technically and commercially viable. This should be distinguished from the approach known as 'activity testing' which is the observation of users for the purpose of fine-tuning an existing technology capability in the context of intended use by the technology supply industry. 
The focus of this paper will be based on the user inspired innovation within the same market, in this case Australia. However, the supply side of the industry also is inspired by innovations in other outside markets where it is perceived there are sufficient similarities.

\section{SMS - A MACRO CASE STUDY}

The Short Message Service (SMS) is an integral part of the digital mobile technology GSM that was launched in 1992 in Europe but now extends over most of the world. Prior to the GSM based mobile service, most markets had experience with an analogue mobile service that just offered a mobile voice telephony service. While SMS was originally only a minor "value added feature' of the GSM mobile voice service, similar to the then paging service, it has evolved over the last 10 to 15 years to become a mass communications service in its own right.

In the last 5 years SMS and premium SMS as it is called supports a whole diversity of business applications ranging from managing field staff to enabling television viewers to vote in 'reality TV.' There is no question that SMS, coined the "ugly duckling" of GSM (Trosby 2004) has been an outstanding market success that could not have been predicted. Many business strategy analysts would argue SMS is an example of a "discontinuous" or "disruptive technology" (Christensen 1996) that has changed the communications paradigm. The perspective in this paper is that SMS is a 'continuous' technology but can be viewed as undergoing changes in quite distinctive phases each identified as a spiral of innovation along a continuum but with different characteristics.

The first stage of SMS evolution we would term Phase 0 as this was the pre-launch stage. This included: the specification of the three service elements in Europe in 1987, how these requirements would be incorporated in the GSM architecture through and the launch of commercial services in Australia in 1993 one year after Europe. Some key decisions were taken during this phase by visionary engineers with experience of data communications in the fixed telecommunications network. The expectation by most outside this inner group was that FAX and circuit switched data were the significant non-voice services to be concerned about. Phase 0 corresponds to the 'onset' of innovation (Utterback and Afuah 1995) where there is great uncertainty about the product and the market.

Phase 1 of the SMS service or the first turn of the spiral of innovation in Australia began in 1992 with the launch of the first commercial service. This phase can be categorised as the 'technology innovation only' phase. SMS was positioned as similar to the existing product, the paging service, but 
being differentiated by its integration with the mobile phone ${ }^{1}$. SMS was an alternative to diversion to voice mail ${ }^{2}$ for digital mobile phones. Mobile phones initially in this phase did not support two way SMS. While international roaming was a key feature of the GSM voice service, SMS international roaming was sporadic due to the incompatibility of the SMS Service Centres. Thus SMS technology in this phase was well developed and stable but not marketed and not generally adopted in the market. By the end of this phase in 1996, two way SMS and international SMS to support international roaming were in place, but SMS messaging between users using mobiles on different networks was not supported by the carriers. SMS messages could however be sent between networks via the internet developed by innovation third players but could not be charged for! This restriction from a user's perspective was a significant usage barrier to SMS being used as a distinct messaging platform rather than just an adjunct to the voice service.

Phase 2 of the SMS evolution is where SMS exploded as a service in its own right. This we would argue was because of strong feedback in the innovation spiral from users feeding ongoing technology innovation. This explosion was ignited first in 1996 by the introduction of the pre-paid mobile service in Italy which in turn influenced the operators in Europe and Australia. Pre-paid technology removed the significant barrier to the development of the youth market that had been deterred from entering the market, by credit controls and budget concerns. However, the current billing system could not charge users for SMS and inter network SMS. Thus phase 2 was a key process innovation enabling growth in what, till then, was a SMS service product innovation as described in the innovation literature.

Thus this phase of intense user and technolegy innovation saw several key market and technology driver elements converge:

- Mobile youth hungry to communicate cost effectively became the new market driver with pre-paid mobile phones

- The initial billing system's inability to charge for many SMS messages from pre-paid mobile phones or where inter network SMS meant that SMS at zero charge was VERY cost effective for this new youth market

- The introduction of 'predictive text' messaging which increased the SMS usage per user significantly ${ }^{3}$

This was a boom period for SMS was characterised by both 'user innovation' and 'technology innovation' as operators quickly implemented effective charging for SMS messaging from both pre-paid and inter-network

\footnotetext{
${ }^{1}$ In the US it was common for users to carry a pager as well as a mobile phone because of the 'mobile party pays' charging principle which was unlike most of the world.

${ }^{2}$ Diversion to voice mail was very common for the previous analogue mobile service.

${ }^{3}$ One estimate is the 'predictive text' increased usage by $30 \%$ per user
} 
SMS mobiles. In Figure 3.1 shows the dramatic increase in SMS traffic when it was marketed to the youth market at zero charge for pre-paid mobile originated SMS. When charging was implemented, usage dropped but when inter-network SMS was activated with charging, the SMS traffic started to climb dramatically. The 'network externality value' to users of being able to SMS anyone irrespective of the network provider far exceeded the SMS charge of 20c per message.

\section{SMS Volumes per Week}

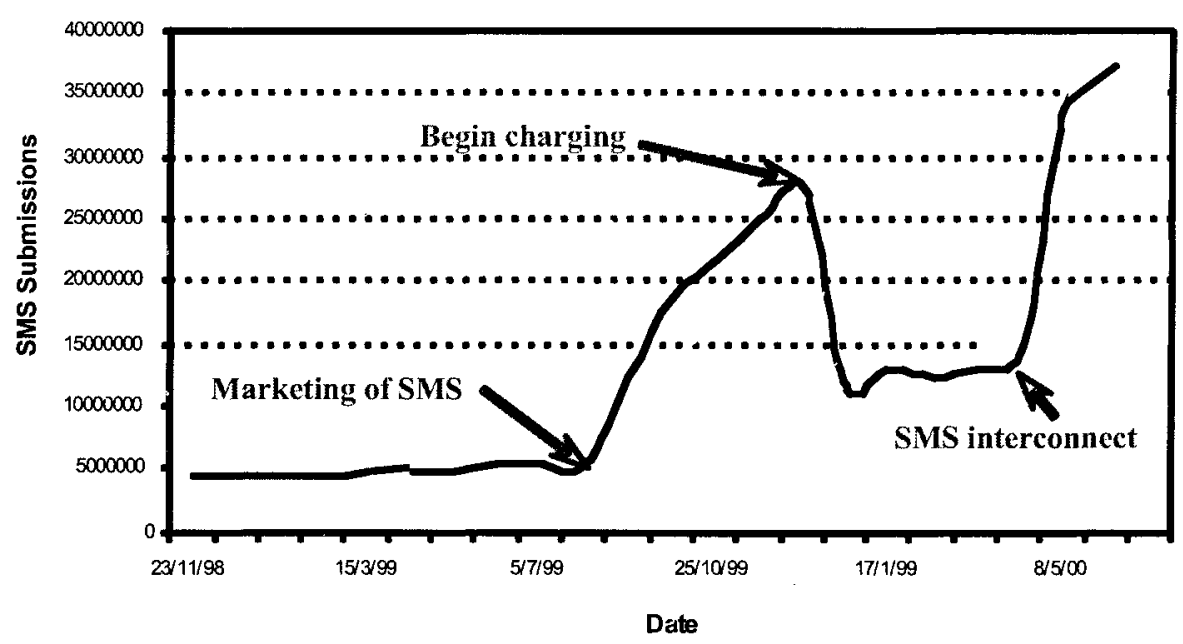

Figure 1. Impact of Inter-network Connection on SMS Traffic in Australia

During the rest of this phase operators did not lower their charges for SMS as they were having extreme difficulty coping with the traffic volumes. The figure shows that the volume increased by an order of magnitude in some 18 months with greatly increased usage per user. This growth in SMS in Australia is shown in Figure 3.2.

By the end of this phase SMS had become a phenomenon particularly associated with youth but its appeal across age demographics had begun and operators were beginning to have to reduce SMS charging as it became a commodity. Since SMS messaging was transparent to the users network operator, operators began competing on the price of SMS messaging. Phase 3 was primarily user inspired innovation by youth consumer users 
appropriating SMS for their use so SMS became a new cost effective communications cultural phenomenon. Research in Japan illustrates the insights from an anthropological perspective of the market adoption of messaging services (Ito 2003).

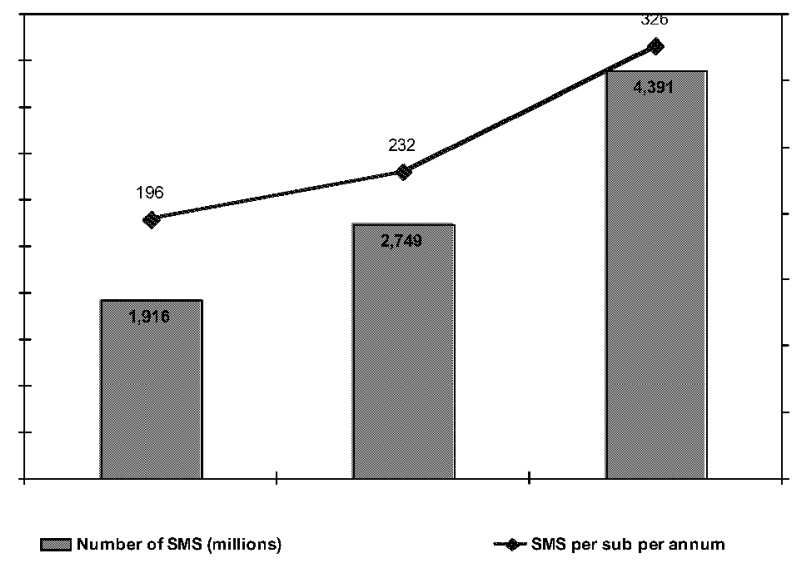

Figure 2. Growth of SMS in Phase 2 in Australia

As this phase in SMS development paralleled the spectacular growth of the internet, attempts by the industry to foist the promise of a "mobile internet" on users with the introduction of Wireless Application Protocol (WAP) was generally regarded as a failure. Another example naively compared to SMS is MMS (Multimedia Service). While cameras integrated into mobile phones has been extremely popular, MMS has been very poorly received generally by the market as it is very different to SMS from a user perspective and has been poorly marketed. Developing a new value proposition linking the power of the internet in the user context of mobility requires a user centred framework focused on developing successive valued applications in evolved phases. We would argue i-Mode developed by NTT 
DoCoMo in Japan in this same period was a success because it focused on market innovation around applications rather than on technology innovation. The i-Mode model presents a total value proposition to the market and not a technology features jig saw puzzle. Important in the overall $\mathrm{i}$-Mode business model was a viable business model for content developers which was not the case for WAP model in most countries

Phase 3 for the SMS innovation spiral started in Australia in about 2001 the overall technology had matured so that it included the useability of SMS on phones as well as the network technologies of billing and robust service centres. SMS pricing from operators was falling enabling SMS to become a platform for messaging applications by business. This phase is characterised by a combination of technology innovation and user innovation but within the business sector. Users in this context are intermediate users or a business. With reduced pricing, particularly for wholesale purchase as a result of the commoditisation of SMS in the previous phase, market innovation in the financial, retail and business service sectors started to flourish and still is expanding. The introduction of premium SMS at the end of Phase 2 with abbreviated numbers enabled the growth of interactive television for voting and audience participation which raised the broad profile of SMS as a powerful platform for the broader consumer and small business market for Phase 3.

To enable business to exploit SMS functionality for what are termed 'vertical' applications ${ }^{4}$, technology innovations have been developed to enable PC based SMS messaging rather than on mobiles and for converting Email to SMS and vice versa.

The key quiestion for the industry in relation to SMS is whether a fourth phase is on the horizon in which SMS is just a 'messaging module' in a variety of new multimedia services types and what will be the its relationship with GPRS based messaging.

\section{UNDERSTANDING USER INNOVATION}

In the previous sections of this paper the concept that users of technology can reinterpret the use of that technology in a way that the industry did not anticipate has been developed with reference to the history of the short messaging service (SMS) on mobile phones. This, it is observed, spurred responses from the industry, albeit rather belatedly, at various stages in the evolution of SMS to develop and refine the technological and commercial means of providing that capability. In this way it is postulated that users are

\footnotetext{
${ }^{4}$ Vertical applications are sectors like education, reality TV etc
} 
functioning as innovators - inspiring innovation, which leads to different approaches in marketing or the modification of the product ${ }^{5}$.

The history of SMS suggests that this process happens at various phases in the evolution of the use of a technological platform, often, but not always $^{6}$, promoting an innovative technological response in one part of the industry or another. This process, it was observed, often required certain other preconditions to be present so did not necessarily happen in a predictable or uniform way. In this spiral of innovation the user is doing much more than just stimulating demand for a new service. They are actually suggesting a different way to use an existing technology platform thus creating new technology?

The history of SMS, interpreted within this conceptual framework, demonstrates the value of understanding user inspired innovation for those involved in designing, producing and marketing technology. It illustrates very well how the technology driven innovation process at any one point can fail to take up or identify a technology that is potentially valued and meaningful to a substantial body of users and that furthermore, conventional market forecasting methods can fail to identify the latent potential in the user population. We contend that this, in part, relates to the conventional way in which users are involved in product design and development and market research.

(Bar and Riis 2000) suggest that conventional industry-led technology design, development and market forecasting are overly reliant on lead users. They say such reliance reinforces the status quo in product development as lead users "tend to be more sophisticated in their understanding of a technology so tend to suggest improvement in the technology itself" (ibid). This focus en the high technology end of the market can result in the locking out of other consumers as the prodict development process "tends to become a professionalised activity" that relegates lay users to the role of a passive consumer (ibid). Furthermore this reliance can lead to "unsatisfactory innovations" and "deprives producers of the insights lay users may have generated".(ibid). They explain lay users ${ }^{8}$ included in the technology innovation process are less constrained by established practice and that "ensuring that the broad lay base... gets access to advanced technology in the early phases of the innovation cycle allows the rate of experimentation at all levels to increase" (ibid).

\footnotetext{
${ }^{5}$ The term product here is taken to include services

${ }^{6}$ This is where the user led innovation opportunity can be lost

${ }^{7}$ A broad definition of technology is used here to encompass any new way of doing something in this case on a telecommunications platform.

${ }^{8}$ Lay users are users without expertise or professional training in a particular field
} 
This part of the paper will examine the role of lay users in user innovation with reference to three user research studies done by the authors (Coutts 1998, 2002, 2003). The primary objective of the studies was to gain insights into the likely adoption of mobile communications technologies of which users had no prior experience. The work has provided insights into the role users play in the innovation process by finding new ways of using the product (i.e. innovating).

From the work we have identified the necessary preconditions for fostering user innovation involving the wider lay user population. (Coutts 2002 , 2003). We suggest that adoption of the technology is part of the innovation process as users have to first appropriate the technology before they can be creative with it - or innovate. Both processes are phenomenological and therefore the authors suggest a qualitative research approach is required which involves "focusing on understanding the nature of the phenomena and their meaning, rather than the incidences.

To understand this phenomenon and its application, a new research methodology is needed which is effective in elucidating responses from target user groups who have had little first hand experience or even awareness of the technological capabilities being explored.

From a range of qualitative research tools, the authors developed a user research methodology which through one encounter with lay users would create the necessary preconditions for embracing cutting edge communications technologies. These technologies were presented to the users in a 'hands on' demonstration by the technologist in the research team. User participants would have to first reach a level of comfort, confidence and familiarity in order to adopt the proposed technological capabilities being presented to them. The research team were able to be reflective about their research assumptions and methodology. This was particularly important for the technologist who was in a sense acting as a proxy for supply industry. The researchers employed a data collection method that captured the subtle attitudinal changes and insights that the users were expressing through this experience and the analysis method allowed the 'meanings' to emerge from out of the data ${ }^{10}$.

Adopting the paradigms of the social sciences the research team postulated that the research methodology had to have a number of elements. It had to have the capacity to engage users in an exploration of the technology in the context of their real life experiences and culture. Since adoption decisions by users (as with all human behaviour) do not reside in the rationale domain only, the researchers needed to also capture and

\footnotetext{
Association

of

Qualitative

Research

(AQR)

http://www.aqr.org.uk/glossary/index.shtml?qualmktres

${ }^{10}$ Grounded theory
} 
understand the perceptions and misperceptions users held about communications technology. This was particularly relevant because the target user group were not technologically savvy and included those elements of the population of lay users who are technologically resistant. Though this was not deliberately prescribed, the selection criteria did seek users with only basic experience of mobile telephony and computer technology. Since the user participants had no experiential reference point, the enquiry method fostered the users' understanding of, and comfort with, the more advanced mobile technological concepts and capabilities. Finally the research approach provided a stimulus to visualising potential utility of these future technologies with which they had no prior experience. User innovation involves a paradigm shift for both the users and producers through an iterative process. The research team not only acted as observers and facilitators but also as participants in the process. The research activity therefore had to provide an effective means of facilitating a change process in a short time frame. The data collected had to reflect the subtle changes taking place and this achieved through the rich text of their conversations and actions.

Focus groups have the required characteristics (Gibbs 1997) and were used as the medium for interaction with users and with elements of ethnography ${ }^{11}$, anthropology, action research, action learning and grounded theory analysis informing the design and conduct of the focus groups.

The research team needed to understand the nature of the users' daily lives, the culture in which they lived and its imperatives in order to understand the context in which the technology would be of value and the form that value - elements of anthropological enquiry. To understand the barriers and enablers to adoption and potential innovation, the researchers needed to 'hear' the values the users bold, the beliefs and perceptions the users have about the technology and themselves, their capabilities and their needs - the elements of ethnography. In effect, the focus group had to simulate an accelerated process of adoption and in doing so provide the impetus for innovation. To take the users through the process of change, the research team used elements of action learning (Yong and Pauleen 2004). A grounded theory approach was used for data analysis.

The results of each project provided insights, at a particular point in time in the evolution of mobile technologies, into how lay users can inspire innovation and coming, as it were, out of left field act as a driver within the innovation spiral. The first project (Coutts 1998) was specifically aimed at a significant market sector that had failed to embrace the opportunities presented by mobile technology at the time. In the second (Coutts 2002) and

${ }^{11}$ Ethnography produces in-depth understanding of real-world social processes investigating the relationship between beliefs and action in social situations.(Forsythe 2001) 
third research projects (Coutts 2003) the focus was on users' responses to future capabilities of digital wireless data technologies.

In the first research project (Coutts 1998) the authors demonstrated user innovation at the end of the production cycle. They reported that their research question had been premised on the wrong assumption that the technology needed to be modified. Instead the products (value added mobile services) were found to be little used due to misperceptions held by the target group about how they might meet their service sector needs. At the beginning of the focus groups participants reported that they couldn't see themselves using mobile phones in their work. However, through examination of their organisational context a new value proposition was created. The study concluded that "the marketing message of how to integrate wireless technology into modern life is not clear to many potential users" (Coutts 1998) The outcome was a marketing strategy tailored to the cultural and structural needs of this sector - this was the user inspired innovation.

In their second research project (Coutts 2002) the authors refined the methodology to aid visualisation by users. This work was seminal in understanding of the meaning users give to the mobile phone which was akin to a form of technological embodiment or 'extension of self'. Because of the very personal relationship users have with their mobile phone (in contrast to their PC) the findings suggested that the imperus for innovative developments in m-commerce applications would come from mobile technology use rather than experience with e-commerce.

The third project (Coutts 2003) explored user reaction to emerging multimedia mobile services for lay users but was segmented on the basis of age, gender and socio-economic status The results indicated the basis for what we have termed a phase shift in tre:innovation spiral as the female users saw media (both stills and video) transforming the nature of the value proposition. However, the meaning or value proposition for the professional sector was around efficiency gains in essential communications and time management ${ }^{12}$.

In all three studies, users reported that from initially seeing little value in the technology being presented they progressed to being able to envisage future use for the technology for themselves ${ }^{13}$. This phenomenon is a key element in the creative process that precedes innovation.

${ }^{12}$ Features including diary and contact lists for example that are synchronised with the office data.

${ }^{13}$ Note we would stress these were lay users, most of whom would not be regarded as early innovators in terms of technology adoption. 
Our review of the three studies demonstrate that the research methodology was effective in revealing the nature of user innovation namely:

- innovation should be viewed as a phenomenon

- changed thinking is required for innovation

- old meanings held by users about the technology need to be understood and new meanings (or value propositions) have to be found

- understanding the necessary preconditions for innovation includes context of use and perceptions of value

The user research methodology simulated the adoption process which led to some users in the groups to appropriate the technology and so inspiring innovation. The presence of a technologist in the team of social researchers helped users to find value in the technology within their own context and translate this into potential product innovation.

This review of the three studies contributes to an understanding of the phenomenon of user innovation. To willing listeners it serves to inform those involved in technology design and development on how they can more easily capture the potential of this dynamic so that they are less likely to miss the opportunities presented by the 'SMSs' of the future.

\section{DISCUSSION AND CONCLUSIONS}

Technology innovation takes place through an evolutionary process involving a complex and interdependent interplay of product inmovation and market diffusion. We have described a theoretical franework for considering this evolutionary process in distinct phases corresponding to the changes in the economic environment, mariset conditions and the state of the product. The argument developed in this paper is that new technologies or products that are spectacularly 'successful' are those that give rise to a spiral driven by the interaction of product innovation and market innovation in at least one of the phases of the product evolution.

This theoretical framework is explored in the context of one of the spectacularly successful new technologies, the SMS service product part of the GSM digital mobile technology. The case history identifies four distinct phases in the evolution of SMS from specification in phase 0 , its modest beginnings in the market in phase 1 to the spectacular growth to a mass phenomenon in phase 2. In phase 3 where SMS is a commodity, it has now become a messaging building block for business messaging services. This study is a macro view of the phases of the innovation spiral throughout product evolution that demonstrated that there is a self-reinforcing process of product and user innovation. 
A closer examination of the user's role in this innovation process was then made through appraisal of three 'user centred' research projects conducted by the authors over seven years. The research provides key insights into the lay users' perspectives and how their appropriation of technology to meet different uses inspires further innovation. All three projects looked at users and their views of mobile technologies including evolving digital services on the mobile platform. A modified focus group methodology was used to engage the lay user community in envisaging how they might use services of which they had no prior knowledge or experience. The research methodology set out was designed to take users through an accelerated process of adaptation and innovation at various phases in the evolution of mobile technologies.

While the user research projects were conducted at different points in time with to the respective product evolution history, they were commonly at the formative phases of the particular products. SMS was not specifically considered in the research as the focus was on emerging products. However, a change in user perspective regarding SMS consistent with the SMS history in Section 3 was observed between the two latter research projects. Such changes in user perspective over time could be effectively captured if incorporated in a longitudinal research process.

We recommend a longitudinal research process involving an annual engagement with a broad lay community of users to uncover the emerging phases of product adoption (and rejection). The focus group format could then be further refined to include part of the session to assess changes in user perception of 'current' products and a second part to consider new products. Such a two part methodology would enable the effective calibration of the phases of product evolution identified and better inform market forecasting, product development and marketing strategies.

We also suggest greater interaction of the research team in the observational room during the sessions using on-line discussion with the mediator and technologist conducting the focus group sessions would improve the user research methodology. While retaining the neutrality of the focus group facilitator, this potential for immediate interaction would enable the research to be more effective to enable testing of emerging insights while still retaining the rich records for reflective consideration.

The overall aim of the user research projects reviewed was to gain key insights into the likely take up of new technologies to enable conventional market research for demand forecasting. In this new innovation framework understands demand is understood in distinct phases that need to be identified. Therefore our future user research will develop the line of questioning for the focus groups and conduct the user sessions with this objective in mind. Further, the proposed more interactive involvement of the 
research team with the focus group facilitator (and technologist) may enable the identification of what the key characteristics of potential different phases of market adoption of new service products might be.

\section{ACKNOWLEDGEMENTS}

The authors acknowledge the financial support from the Smart Internet Cooperative Research Centre (www.smartinternet.com.au ) for the research referenced in the paper up to the end of 2003 and the encouragement of colleagues to commercialize some potential products based on this ìnnovation framework.

\section{References}

Bar and Riis (2000), Tapping User-Driven Innovation: A New Rationale for Universal Service, Information Society; April , Vol.16 Issue 2, p99, 10p

Christensen (1997), The Innovator's Dilemma: When Disruptive Technologies Cause Great Firms to Fail, Clayton Christensen, Harvard Business School Press.

Coutts (1998), 'A User Methodology - Identifying Telecommunications Needs', Pam Coutts, Communications Research Forum (CRF), Canberra, September 24-25.

Coutts, P. (2002) Banking on the move - Characterising user bottlenecks for m-commerce uptake, Communications Research Forum (CRF), Canberra, 2-3 October

Coutts. P., Alport, K., Coutts, R., and Morell, D. (2003),Beyond the Wireless Internet Hype Re-engaging the User, Communications Research Forum, Canberra, 1-2 October

Coutts (2004), An Internet for Transactions: A User Perspective to Inform Future Business Models, Reg Coutts, Pacific Telecommunications Conference (PTC) Hawaii, January

Damsgaard and Gao (2004), A Framework for Analyzing Mobile Telecommunications Market Development, MOBIS'2005, 15-17 September, Oslo, Norway

Forsythe (2001), Studying Those Who Study Us $\Lambda \mathrm{n}$ Anthropologist in the World of Artificial Intelligence, Diana E. Forsythe, Stantord University Press.

Gibbs, A.(1997), Focus groups", Social Research Update, 'ssue 19, University of Surrey.

Ito, M. and Okabe, D. (2003), Mobile Phones, Japanese Youth, and the Replacement of Social Contact, Unpublished.

Trosby, F. (2004), SMS, the strange duckling of GSM, Telektronikk 3.

Utterback, J. M. and Afuah, A. N. (1995), The Dynamic "Diamond": A Technological Innovation perspective, The International Centre for Research on the Management of Technology, MIT, November

Yong, P. and Pauleen, d. (2004), Generating and Analysing Data for Applied Research on Emerging Technologies: a Grounded Action Learning Approach, Information Research, Vol. 9 No. 4, July 